\title{
Improving Switch Lowering for The LLVM Compiler System
}

\author{
Anton Korobeynikov \\ Saint Petersburg State University \\ Email: asl@math.spbu.ru
}

\begin{abstract}
Switch-case statements (or switches) provide a natural way to express multiway branching control flow semantics. They are common in many applications including compilers, parsers, text processing programs, virtual machines. Various optimizations for switches has been studied for many years. This paper presents the description of switch lowering refactoring recently made for the LLVM Compiler System [1].
\end{abstract}

\section{INTRODUCTION}

Many imperative high-level programming languages offer a form of multiway branch statement. The examples are switch statement in $\mathrm{C} / \mathrm{C}++/ J a v a$, case in Pascal, etc. Switches can be usually seen in many sophisticated programs like compilers, virtual machines, parsers because these applications often use some value to select an action among a large collection of possible. Almost all finite state machines implementations uses switches in order to translate from one state to another. Such FSMs, for example, are generated on-the-fly in applications doing any sort of pattern matching.

This is compiler task to examine the switch and emit the most effective code for this platfrom to implement multiway branches. The possible issues involved in producing such code are non-trivial. The main tradeof is, as usual, size-speed. However, in most real-world cases small size of generated code for multiway branch facility implies its big speed, since switches are often used in the tight inner loops of the application.

This paper presents description of the switch lowering refactoring made in the LLVM Compiler System recently. Section $\Pi$ iI introduces briefly to the LLVM itself, its goals, advantages and fields of use. Section III is devoted to the miscellaneous strategies of switch emission and the concrete description of the LLVM switch lowering algorithm.

\section{THE LLVM COMPILER SySTEM}

The LLVM Compiler System was originally designed as a compiler framework to support transparent, lifelong program analysis and transformation for arbitrary programs, by providing high-level information to compiler transformations at compile-time, link-time, run-time and in the idle time between runs [2].

Currently the key features of LLVM are:

- Has many built-in sophisticated optimization algorithms.

- Can be used as a JIT compiler.

- It is retargettable, supports many targets.

- Has Link Time Optimization (LTO) support.
- Can be successfully used as a drop-in replacement for GCC.

The LLVM code representation describes a program using an abstract RISC-like instruction set but with key higherlevel information for effective analysis. This includes type information, explicit control flow graphs, and an explicit dataflow representation (using an infinite, typed register set in Static Single Assignment form) [3]. LLVM provides equivalent textual, binary and in-memory code representation.

Let's see, how LLVM code looks like. Consider the naive recursive function, which calculates the Fibonacci sequence:

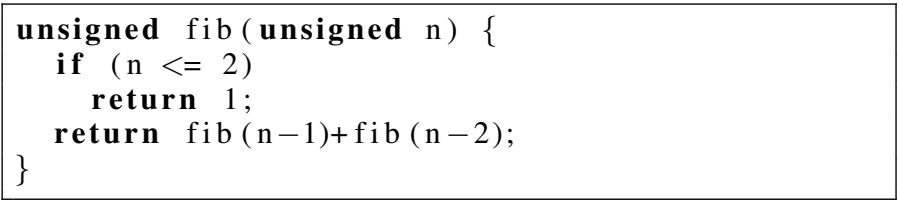

The corresponding LLVM code is:

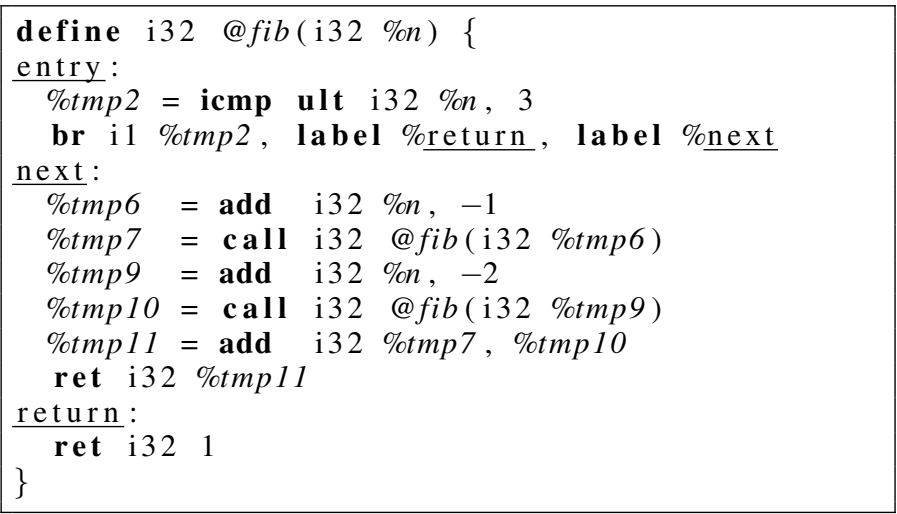

One can see here some of LLVM's key features including embedded type information and SSA form of the representation. LLVM's built-in optimization passes can detect tail recursion in this case and optimize it out.

All optimizations can be performed in LLVM in two ways:

1) As LLVM-to-LLVM pass taking LLVM code representation as input and producing LLVM code as output.

2) During codegeneration for target machine.

The second approach is more suitable for our work, since it allows us to use some target machine information, resulting to much better code. We cannot perform well with LLVMto-LLVM pass, since LLVM representation is target machine independent. 
The LLVM target-independent code generator is a framework that provides a suite of reusable components for translating the LLVM internal representation to the machine code for a specified target - either in assembly form (suitable for a static compiler) or in binary machine code format (usable for a JIT compiler). The LLVM target-independent code generator consists of five main components:

1) Abstract target description interfaces which capture important properties about various aspects of the machine, independently of how they will be used.

2) Classes used to represent the machine code being generated for a target. These classes are intended to be abstract enough to represent the machine code for any target machine.

3) Target-independent algorithms used to implement various phases of native code generation (register allocation, scheduling, stack frame representation, etc). Switch lowering is also implemented here.

4) Implementations of the abstract target description interfaces for particular targets. These machine descriptions make use of the components provided by LLVM, and can optionally provide custom target-specific passes, to build complete code generators for a specific target.

5) The target-independent JIT components. The LLVM JIT is completely target independent (it uses special structure to interface for target-specific issues).

Lowering is the process of turning one instruction (switch in our case) into series of more low-level instructions. This process surely depends on the target machine and the switch itself.

\section{SWITCH LOWERING IMPLEMENTATION}

Switches are represented in the LLVM with the help of switch instruction. The syntax for this instruction is:

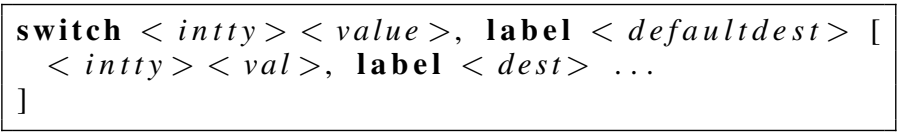

The switch instruction uses three parameters: an integer comparison value value, a default label destination, and an array of pairs of comparison value constants and label's. The table is not allowed to contain duplicate constant entries.

Some examples will follow.

; Emulate a conditional br instruction
$\%$ Val = zext i1 \%value to i32
switch i32\%Val, label \%truedest
i32 0, label \%
]
; Emulate an unconditional br instruction
switch i32 0, label \%
; Implement [ ] jump table:
switch i32\%val, label \%otherwise [
i32 0, label \%onzero
i32 1, label \%onone
i32 2, label \%ontwo
]

Consider the following switch:

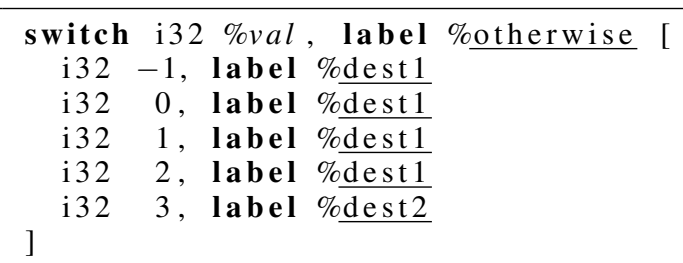

One can see, that cases cases 0-2 goes to one destination and case 3 - to another. So, we can "compact" the switch writing such LLVM "pseudocode":

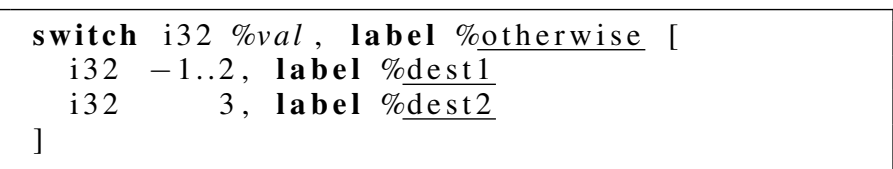

We call this "pseudocode", because LLVM does not support switches with "compact" case ranges (like gcc trees, for example) now. The main advantage of this representation is that we can go to label dest 1 just emitting range comparison (so do only 2 comparisons) and not comparison per each case entry. Internally we will operate on switches in this form. Also we are assuming that cases are sorted withing switch.

This source will be used later as a source switch being lowered. It will be called "example switch" everywhere, otherwise explicitly mentioned.

We will use different metrics to examine switch. Two of them are:

1) Switch density. The definition is straightforward.

2) Number of comparisons needed to emit all cases. This is just sum of number of comparisons needed to emit each switch case. One comparison is needed to emit single case, two - for case range.

\section{A. Different Strategies to Lower Switch}

1) Small Switches: All other strategies involve some overhead needed to prepare switch data for future use. This overhead can be significant for small switches. We consider (compacted) switch to be small, if it contains 3 or less cases. In this case we're just emitting series of branches. Also note, even we are saying, that case range uses two comparisons, actually, we need only one unsigned comparison plus some additional arithmetical operations. This "trick" is shown below during lowering of our example switch.

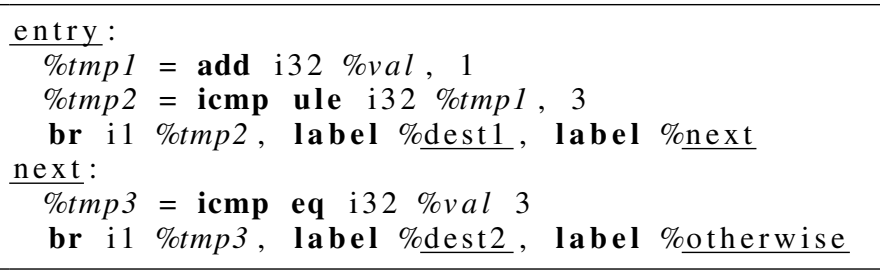

This strategy is just a linear search over all cases. So, its runtime is linear on number of cases. 
TABLE I

Example CASE Set

\begin{tabular}{c|c}
\hline Case & Binary \\
\hline 0 & $00000000_{2}$ \\
\hline 1 & $00000001_{2}$ \\
\hline 129 & $10000001_{2}$ \\
\hline 131 & $10000011_{2}$ \\
\hline
\end{tabular}

densitites attends maximum at this pivot. Also, it will be better to split switch maximizing the distance between subtrees.

Taking into account all mentioned arguments, we are assigning each pivot its own quality. Let $l_{i}$ and $r_{i}$ denote the densities of the left and right subtrees being split at pivot $i$. Let $a_{i}$ denote the maximal case of the left subtree and $b_{i}$ minimal case of the right subtree. The quality $q_{i}$ of pivot $i$ is defined as

$$
q_{i}=\left(l_{i}+r_{i}\right) \log \left(b_{i}-a_{i}\right) .
$$

We are selecting pivot with maximum quality. All other details are the same as for ordinary binary tree. Such pivot selection procedure will surely "throw out" trivial cases (splitting in the middle of the dense block) and will prefer more far subtrees, when sum of the densities for two different pivots are close.

Surely, resulting tree is not balanced any more, thus this strategy should be used only in connection with other strategies like jump tables emission.

6) Multiway Radix Search Trees: Multiway radix search trees (or MRST for short) is a novel technique first presented in [4]. In general it is a sort of non-perfect hashing but designed specially for static sparse search trees. This is really important method, since it can turn sparse case set into series of much more denser sets suitable for applying of other strategies listed here. We will cite the description from [4].

The algorithm looks at the input set of cases and finds a short sequence, or window, of consecutive bits that distinguishes the cases into several subsets. The algorithm generates code that branches on the value of the window in the run-time input thus selecting a specific set of candidate cases based on the input value. For empty sets, the branches lead to the default handle; for sets containing one case, to simple comparison of the case and the input value; and for larger sets, to recursive invocations of the algorithm.

It is desirable to find long windows, in order to make the search tree wide and shallow. However, the length of the windows should be limited, since the branches require hash tables that grow exponentially in the window length. The simple greedy strategy of finding the longest critical window that distinguishes the cases into more than a threshold number of subsets relative to the window length is used. In [4] it is proven, that average number of branches for MRST algorithm is at most three.

Consider the table [ We see, that cases $0,1,129$ and 131 are almost uniquely determined by their last two bits. The one exception is the pair 1 and 129 , where bit 7 can determine the case. Therefore we can quickly narrow down the candidate cases for a given run-time input by hashing first on the last two bits and then, if necessary, on bit 7, the leftmost bit. Finally, we can compare the input value with the unique remaining candidate case. Note that the code should branch directly to the default handler for any run-time input ending in $10_{2}$ since no case ends in this bit pattern.

MRST tends to be global switch rewriting strategy. That is why it is hard to use it "stacked" with other methods. Consider the switch with two dense case sets separated far away. In most cases, it will be better to split such switch into two case ranges and emit two jump tables, not to apply MRST.

\section{B. Switch Lowering}

Before our work LLVM's switch lowering algorithm was simple: at first it tried to emit jump table, if the switch had been dense enough. Otherwise the algorithm just emitted balanced binary search tree. Also, algorithm operated on the switch itself, not its "compacted" form. This leads to inefficient code in many cases.

Current LLVM switch lowering algorithm is simple worklist-driven algorithm trying to apply different strategies for case set popped from the worklist.

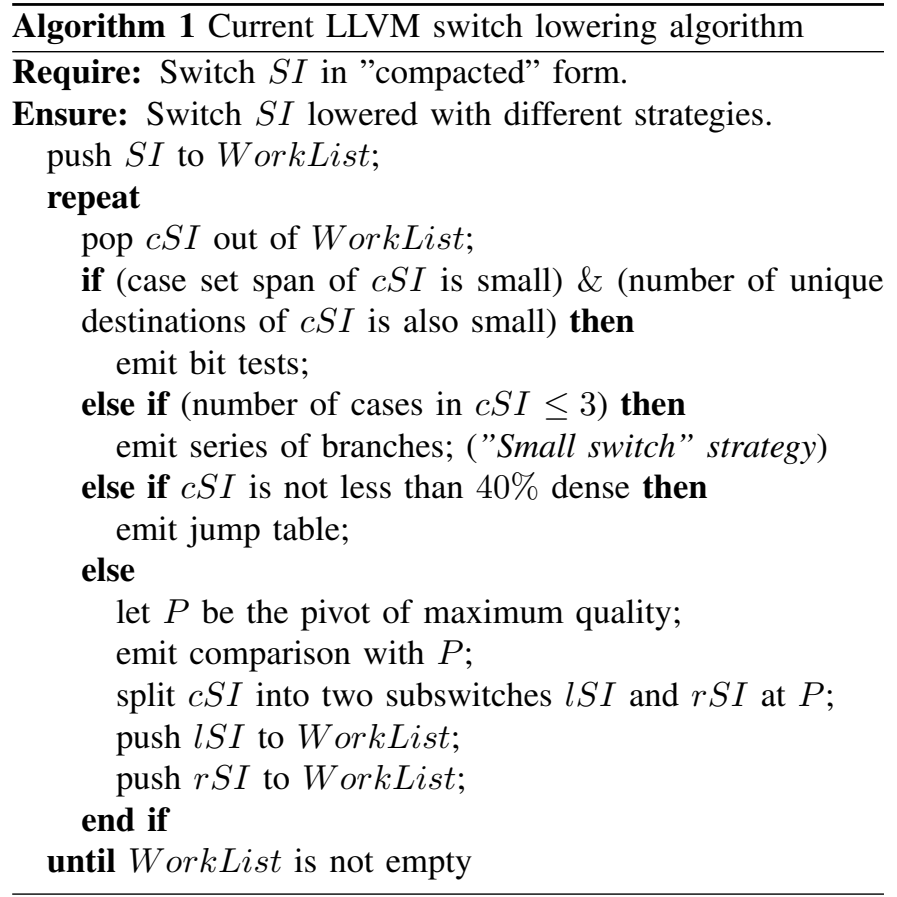

\section{RELATED WORK}

There have been a few papers discussing the issues arising in optimizations of switches.

Different methods were studied by Bernstein in [5] including linear search, binary search and jump tables. He found, that linear search is faster than the jump table for small switches (when number of cases is 3 or less) and that binary search is faster than linear search for big switches. The methods of breaking big switch into smaller and denser clusters were also examined. He proposed, that optimal solution of the 
TABLE II

COMPILATION TIMES

\begin{tabular}{c||c|c}
\hline Case & Old switch lowering & New switch lowering \\
\hline Mozilla Suite & $37^{m} 47^{s}$ & $35^{m} 21^{s}$ \\
\hline Qt Library & $28^{m} 54^{s}$ & $27^{m} 01^{s}$ \\
\hline
\end{tabular}

clustering problem is NP-complete. However, in [6] Kannan and Proebsting presented optimal clustering algorithm, which runs in $O\left(n^{2}\right)$ time.

In [7] switch lowering problem was studied from the position of a static search problem. A summary of the various methods were presented, and these methods were analyzed in terms of time and space complexity of the executable code, and also compilation time. A general algorithm for code generation was presented and was shown to improve Bernstein's algorithm in a number of ways.

MRST algorithm was firstly presented in [4]. Authors discussed other possible switch lowering strategies including linear search, balanced binary tree, combination of binary trees and hash tables, and jump tables in comparison with MRST. Also the implementation of MRST algorithm for $l c c$ compiler was presented.

In [8] the switch lowering problem was examined when there are additional information about the case hit frequency. Authors presented different strategies such as hot case hoisting and switch case partitioning which lead to significant reduction of running time. So, actually, they are studied profiledriven switch lowering.

Some approaches assume that switch cases have been transformed to a series of conditional branches and try to reorder these branches according to their importance [9] or convert them into indirect jumps [10].

\section{Results, CONClusion AND Future Work}

Unfortunately, SPEC testsuite was unavailable for author. That is why we perform comparison in the following way:

- Perform llvm-gcc compiler bootstrap with and without improving switch lowering algorithm.

- Build Mozilla Suite and Trolltech' Qt library with both compilers

- Compare build times.

All tests were performed on dual-core AMD Opteron 2210 workstation with $2 \mathrm{~Gb}$ RAM. Only one core was used for tests. The results are shown in the table $\amalg$

Possible future work is summarized below.

- We haven't added MRST algorithm yet due to problems noted in the section III-A6. Actually, we need to develop some local sparseness metric, which can be used to decide, whether MRST algorithm or other techniques should be run on switch.

- We haven't studied any profile-driven switch lowering. We suppose, that impact from such optimization can be significant.
- It can be interesting to try to implement switch case clustering algorithm from [6] and compare it with another strategies.

\section{ACKNOWLEDGMENT}

Author would like to thank Chris Lattner for the help and many interesting ideas.

\section{REFERENCES}

[1] The LLVM Compiler System. [Online]. Available: http://llvm.org

[2] C. Lattner and V. Adve, "LLVM: A Compilation Framework for Lifelong Program Analysis \& Transformation," in Proceedings of the 2004 International Symposium on Code Generation and Optimization (CGO'04), Palo Alto, California, Mar 2004. [Online]. Available: http://llvm.org/pubs/2004-01-30-CGO-LLVM.html

[3] C. Lattner, "Macroscopic Data Structure Analysis and Optimization," Ph.D. dissertation, Computer Science Dept., University of Illinois at Urbana-Champaign, Urbana, IL, May 2005. [Online]. Available: http://llvm.org/pubs/2005-05-04-LattnerPHDThesis.html

[4] U. Erlingsson, M. S. Krishnamoorthy, and T. V. Raman, "Efficient Multiway Radix Search Trees," Information Processing Letters, vol. 60, no. 3, pp. 115-120, 1996. [Online]. Available: http: //citeseer.ist.psu.edu/erlingsson96efficient.html

[5] R. L. Bernstein, "Producing good code for the case statement," Softw. Pract. Exper, vol. 15, no. 10, pp. 1021-1024, 1985.

[6] S. Kannan and T. Proebsting, "Correction to Producing Good Code for the Case Statement," 1994. [Online]. Available: http: //citeseer.ist.psu.edu/kannan94correction.html

[7] D. A. Spuler, "Compiler code generation for multiway branch statements as a static search problem," Department of Computer Science, James Cook University, Australia, Tech. Rep., January 1994.

[8] P. Zhao and J. N. Amaral, "Feedback-directed switch-case statement optimization," in ICPPW '05: Proceedings of the 2005 International Conference on Parallel Processing Workshops (ICPPW'05). Washington, DC, USA: IEEE Computer Society, 2005, pp. 295-302. [Online]. Available: http://ieeexplore.ieee.org/xpls/abs_all.jsp?arnumber=1488708

[9] M. Yang, G.-R. Uh, and D. B. Whalley, "Efficient and effective branch reordering using profile data," ACM Trans. Program. Lang. Syst., vol. 24, no. 6, pp. 667-697, 2002.

[10] G.-R. Uh and D. B. Whalley, "Effectively exploiting indirect jumps," Software-Practice and Experience, vol. 29, no. 12, pp. 1061-1101, 1999. [Online]. Available: http://citeseer.ist.psu.edu/article/ uh97effectively.html 